\title{
Leucine-rich repeat containing 4 act as an autophagy inhibitor that restores sensitivity of glioblastoma to temozolomide
}

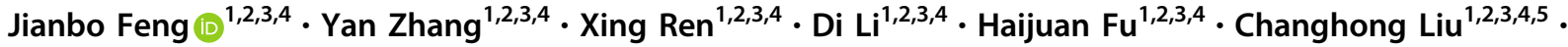 \\ Wen Zhou ${ }^{2,3,4} \cdot$ Qing Liu ${ }^{6}$ Qiang Liu ${ }^{7}$ Minghua $\mathrm{Wu}^{1,2,3,4}$
}

Received: 2 September 2019 / Revised: 14 April 2020 / Accepted: 23 April 2020 / Published online: 5 May 2020

(c) The Author(s) 2020. This article is published with open access

\begin{abstract}
Temozolomide (TMZ) insensitivity and resistance are major causes of treatment failure and poor prognosis for GBM patients. Here, we identify LRRC4 as a novel autophagy inhibitor that restores the sensitivity of GBMs to TMZ. LRRC4 was associated with the DEPTOR/mTOR complex, and this interaction resulted in autophagy inhibition. Further investigation demonstrated that the PDZ binding domain of LRRC4 binds to the PDZ domain of DEPTOR. This binding decreases the half-life of DEPTOR via ubiquitination, thus inhibiting GBM cell autophagy and increasing the TMZ treatment response of GBM. Combined LRRC4 expression and TMZ treatment prolonged the survival of mice with tumour xenografts. Furthermore, the levels of LRRC4, DEPTOR and autophagy are clinically relevant for GBM, indicating that LRRC4 is likely to have significant potential as a therapeutic marker and target for TMZ treatment in glioma patients.
\end{abstract}

\section{Introduction}

Glioblastoma (GBM) is the most common malignant primary brain tumour in adults and one of the most lethal

Supplementary information The online version of this article (https:// doi.org/10.1038/s41388-020-1312-6) contains supplementary material, which is available to authorized users.

Minghua $\mathrm{Wu}$

wuminghua554@aliyun.com

1 Hunan Provincial Tumor Hospital and the Affiliated Tumor Hospital of Xiangya Medical School, Central South University, Changsha 410006 Hunan, China

2 Cancer Research Institute, School of Basic Medical Science, Central South University, Changsha 410078 Hunan, China

3 Key Laboratory of Carcinogenesis and Cancer Invasion, Ministry of Education, Changsha 410078 Hunan, China

4 Key Laboratory of Carcinogenesis, Ministry of Health, Changsha 410078 Hunan, China

5 Institute of Medical Sciences, The Second Hospital of Shandong University, Jinan 250033 Shandong, China

6 Xiangya Hospital, Central South University, Changsha 410008 Hunan, China

7 Third Xiangya Hospital, Central South University, Changsha 410013 Hunan, China human cancers [1], with an average survival of slightly over 1 year after the initial diagnosis [2]. Diffuse invasion of tumour cells into surrounding brain tissue is a common characteristic of malignant glioma and is responsible for treatment failure or relapse even when maximal surgical resection is performed [3]. Moreover, GBM is among the most resistant cancer types to radiation and cytotoxic chemotherapy [4]. Although temozolomide (TMZ) has been clinically shown to prolong GBM patient survival, the treatment outcome is frequently dependent on O6methylguanine-DNA methyltransferase (MGMT) expression. In glioma, patient sensitivity to TMZ and the associated enhanced overall survival are frequently linked to low MGMT expression and increased MGMT promoter methylation [5]. However, the TMZ resistance phenomenon can still occur, and clinical trials that aiming to overcome TMZ resistance by exploring the modulation of MGMT or the addition of targeted agents cannot efficiently improve treatment efficacy [6]. Thus, a deeper understanding of the mechanisms contributing to TMZ resistance could provide prospective molecular targets for glioma therapy.

Autophagy is an evolutionarily conserved catabolic process that involves the sequestration and transport of damaged organelles and misfolded and dysfunctional proteins to the lysosomes for degradation [7]. Following lysosomal degradation, cells restore the intracellular nutrient supply during starvation and protect the cell against 
damage caused by toxic macromolecules and damaged organelles [8, 9]. In addition to nutrient status and intracellular damage, environmental stressors such as hypoxia [10], ER stress [11] and reactive oxygen species accumulation [12] can also induce autophagy. Normal autophagy plays important physiological roles in human health, and abnormal autophagy leads to various diseases, such as neurodegenerative disorders [13] and cancer progression [14]. The role of autophagy differs in different stages of cancer development; initially, autophagy likely has a preventive effect against cancer, but once a tumour develops, the cancer cells could utilize autophagy for their own cytoprotection [15]. It seems that autophagy plays dual roles in cancer progression. Appropriate autophagy acts as a cytoprotective mechanism leading to tumour cell apoptosis resistance, however, excessive autophagy promotes tumour cell death. Recent studies have emphasized the role of autophagy in tumour promotion [16] and drug resistance [17], and inhibiting autophagy significantly increased chemotherapy efficacy [18]; however, the underlying mechanism connecting autophagy and drug resistance is not clear. It is of paramount importance to elucidate the mechanism of autophagy in chemotherapy resistance.

Leucine-rich repeat containing 4 (LRRC4), also called netrin-G ligand-2, is a member of the leucine-rich repeat (LRR) superfamily [19]. Previous studies have confirmed that LRRC4 plays a central role in early nervous system development and differentiation, especially during synapse formation [20-22]; in addition, our group first confirmed a new function of LRRC4 as a tumour suppressor for glioma [23, 24]. LRRC4 reduced the activity of the Ras/c-Raf/ERK/MAPK signalling pathways and inhibited GBM cell proliferation and invasion [25, 26]. Mechanistically, LRRC4 abolished ERK1/2 activation and inhibited ERK1/2 nuclear translocation through direct interaction with ERK1/2 and then inhibited ERK1/2 binding to MEK [27]. Moreover, our recent study also found that LRRC4 plays an important role in the GBM immunemicroenvironment. LRRC4 bound to phosphoinositidedependent protein kinase 1 and HSP90 to promote NF-кB translocation and cytokine production in GBM cells and influenced the infiltration of Treg cells in the GBM microenvironment [28].

Here, we first demonstrated that LRRC4 is a novel inhibitor of autophagy. LRRC4 directly interacted with DEPTOR (DEP domain containing mTOR interacting protein), which, as a mTOR inhibitor, bound to mTORC1 and mTORC2 via the PDZ domain. LRRC4 decreased the protein level of DEPTOR, which resulted in mTOR activation, thereby decreasing the cellular autophagy level. LRRC4 inhibited cell autophagy, restoring TMZ treatment sensitivity in GBM, which could be used as a possible therapeutic strategy.

\section{Results}

\section{LRRC4 is negatively associated with autophagy signals and glioma patient outcomes}

Our group have confirmed LRRC4 as a tumour suppressor for glioma by inhibiting GBM cell proliferation and invasion [24-26]. To investigate the new function of LRRC4 in glioma, we used RNA sequencing to analyse the differences in gene expression between LRRC4 stable ectopic expression and control U251 cell lines (Fig. 1a). Cluster profiler package was used to analyze these data, and result from KEGG pathway analysis showed that the differentially regulated genes participate in lysosomal function and drug metabolism (Fig. 1b), suggesting that LRRC4 may be associated with autophagy signalling and drug resistance in glioma. As autophagy plays an important role in drug resistance, we next focus our attention on the correlation between LRRC4 and autophagy pathway. We used the cBioPortal (http://www.cbioportal.org/) to analyse the correlations between LRRC4 and autophagyrelated genes in GBM samples from TCGA database. In Cohort 1 (GBM, TCGA, Cell 2013), LRRC4 was negatively correlated with BECN1 and MAP1LC3B (Fig. 1c), which are genes that contribute to the origination of autolysosome and autophagy maker gene. Consistently, LRRC4 was also negatively correlated with BECN1 and MAP1LC3B in Cohort 2 (GBM Multiforme, TCGA, PanCancer Atlas) (Fig. 1d). We next evaluated the relationship between expression of LRRC4 and glioma survival rate. By using GEPIA (http://gepia.cancer-pku.cn/ index.html), we found that the high expression LRRC4 group was strongly associated with longer survival (Fig. 1e), while BECN1 were seemingly associated with shorter survival $(p=0.051)$ (Fig. 1f). It suggested that glioma patients with high autophagy level have a worse prognosis than those patients with low autophagy level. Taken together, these data indicated that LRRC4 was inversely associated with autophagy signalling and glioma patient outcomes.

\section{LRRC4 inhibits autophagy activation in glioblastoma cells}

How does LRRC4 regulate autophagy? Next, we examined the role of LRRC4 in cells autophagy via some cell biology experiments. LRRC4 is primarily expressed in normal brain tissues whilst lacking in GBM cells and primary glioma cells (Fig. S1). So, we conducted ectopic expression of LRRC4 to proceed with further investigation. Overexpression of LRRC4 decreased the expression of LC3B, a autophagy maker protein, in U251 cells and PG2 cells (Fig. 2a). LRRC4 knockdown restored the 

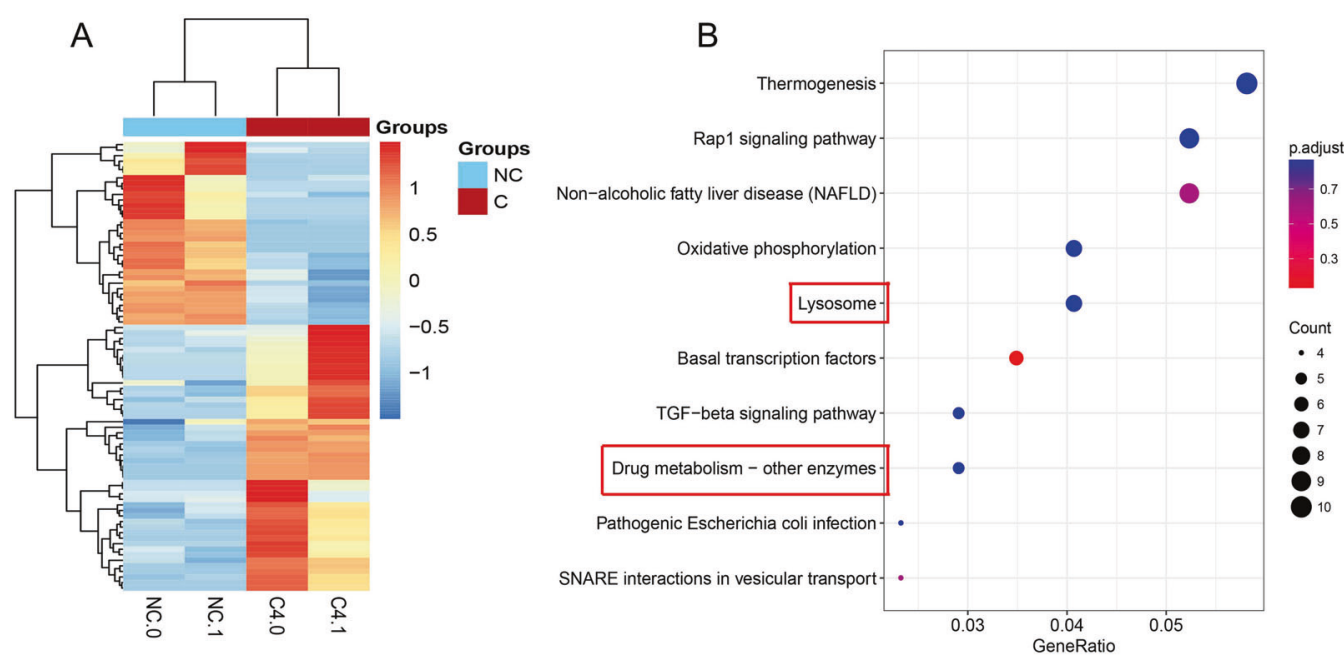

C

Cohort1(Glioblastoma,TCGA,cell 2013)

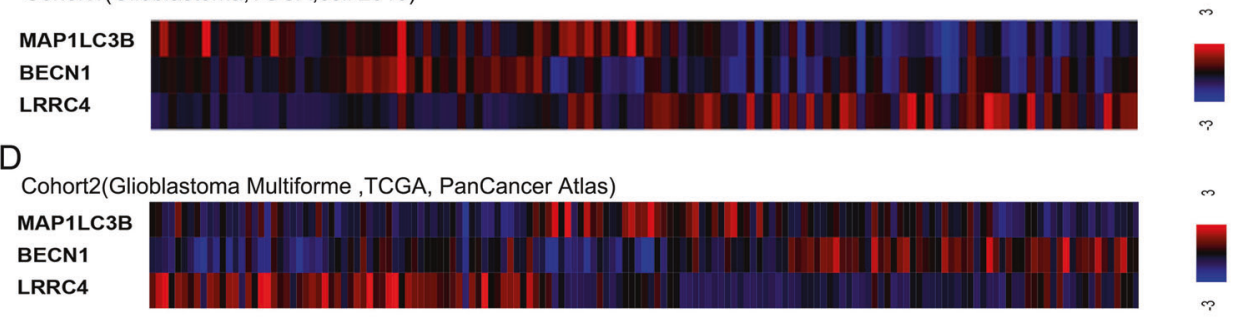

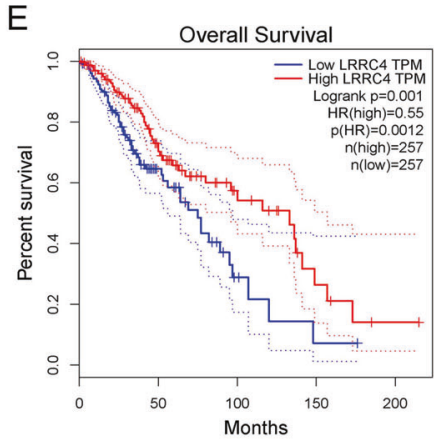

Fig. 1 LRRC4 is negatively associated with autophagy signals and glioma patient outcomes. a Gene expression clustering diagram between $\mathrm{U} 251$ cells (NC: control) and U251 cells that stably expressed LRRC4 (C4: LRRC4). b KEGG pathway enrichment using differentially expressed genes between U251 cells (NC: control) and U251 cells that stably expressed LRRC4 (C4: LRRC4). The gene ratio means the proportion of genes related to this signalling pathway in the

inhibition effect of LRRC4 on autophagy maker protein LC3B in stable LRRC4-overexpressing U251 and PG2 cells (Fig. 2a). To confirm this finding, p3.1-LRRC4 plasmid was transiently transfected into U251 cells at increasing plasmid concentrations. The results showed that LC3II was decreased and p62, which binds ubiquitin and LC3 and is a selective substrate for autophagy, expression was increased in a plasmid concentrationdependent manner (Fig. 2b). Starvation activated cell autophagy has been well described, and we wondered whether the inhibition of autophagy induced by LRRC4

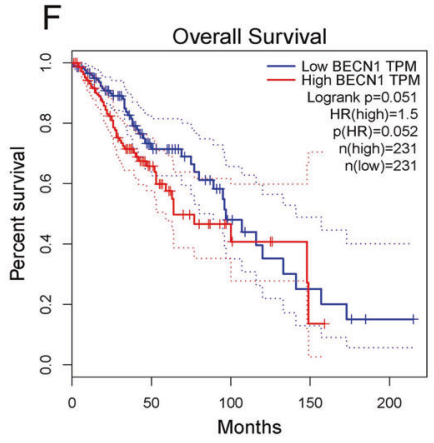

differentially expressed genes. Correlation analysis of LRRC4 and autophagy-related genes MAP1LC3Band BECN1 in Cohort 1 (Glioblastoma, TCGA, Cell 2013) (c) and Cohort 2 (Glioblastoma Multiforme, TCGA, PanCancer Atlas) (d) of GBM samples from TCGA database. Kaplan-Meier curve depicting survival of patients with low and high LRRC4 (e), BECN1 (f) in TCGA.

could be restored. We compared the effect of LRRC4 on autophagy genes under normal conditions and starvation conditions. We found that LRRC4 also inhibited GBM cell autophagy in the starvation state. The results showed that under both conditions, LRRC4 inhibited GBM cell autophagy (Fig. 2c). We established U251 cells and PG2 cells (primary cultured GBM cells) that stably expressed a tandem mRFP-EGFP-LC3 plasmid and found that LRRC4 prevented the autophagic flux in U251 cells and PG2 cells (Fig. 2d). Under starvation conditions, in line with these results, an immunostaining assay also showed that 
A

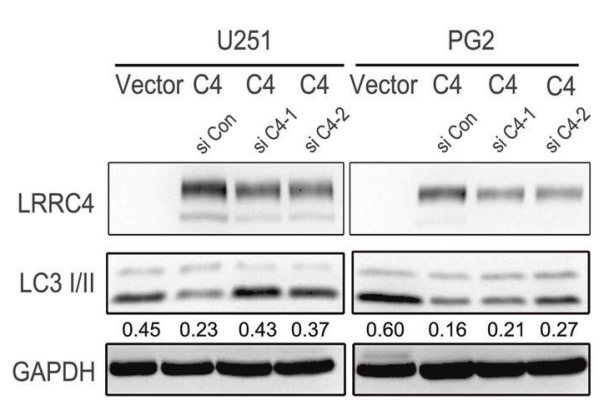

B

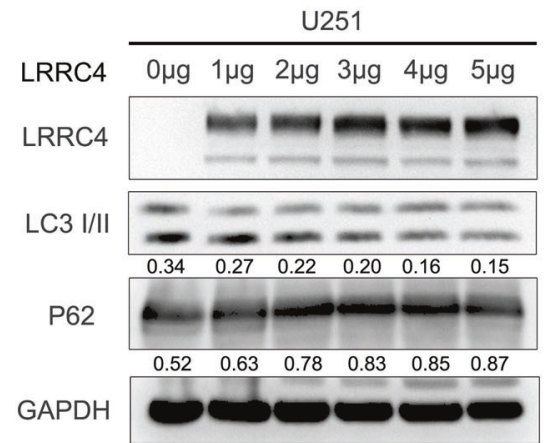

C

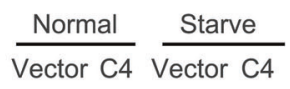

LRRC4

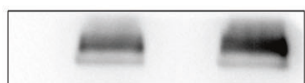

LC3 I/II

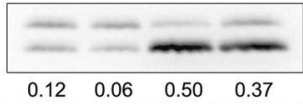

P62

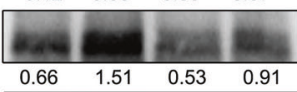

GAPDH
D
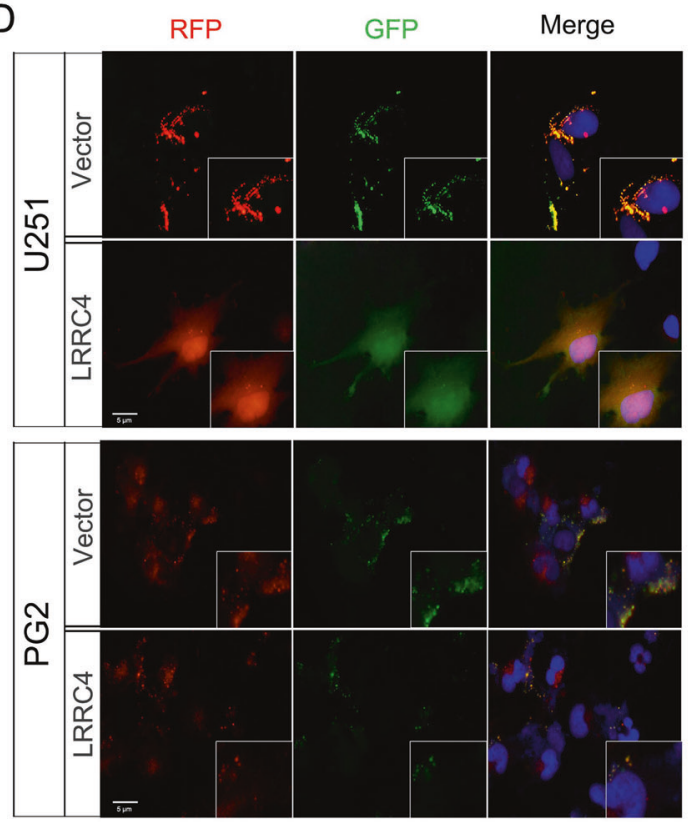

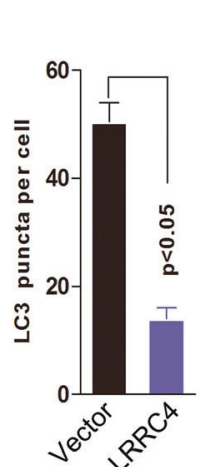

E

Merge
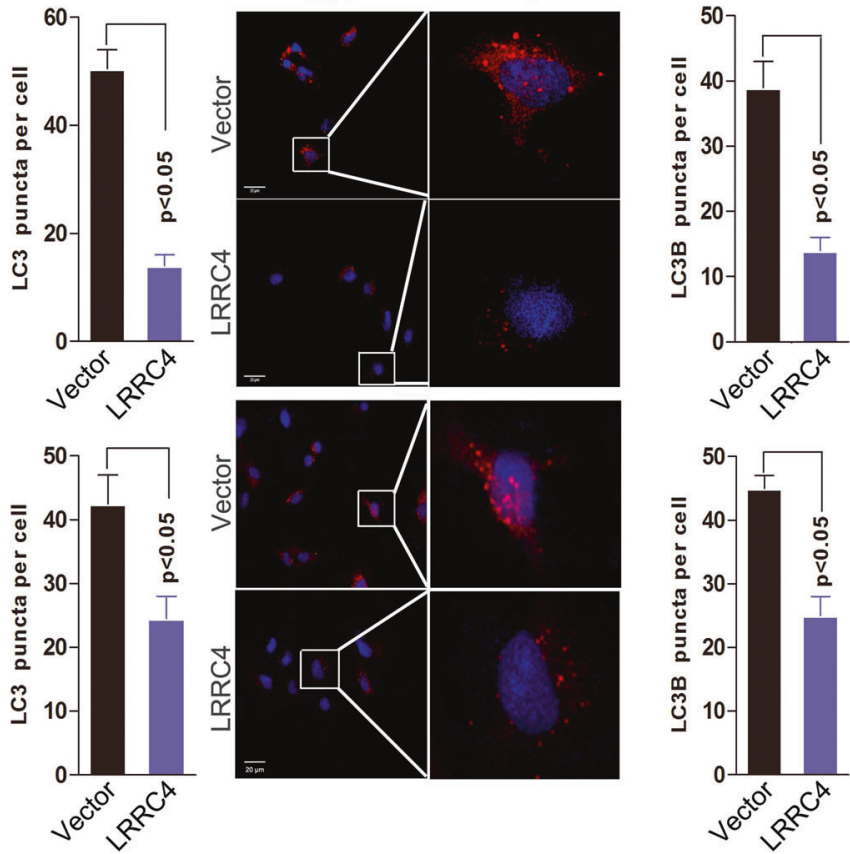

$\mathrm{F}$
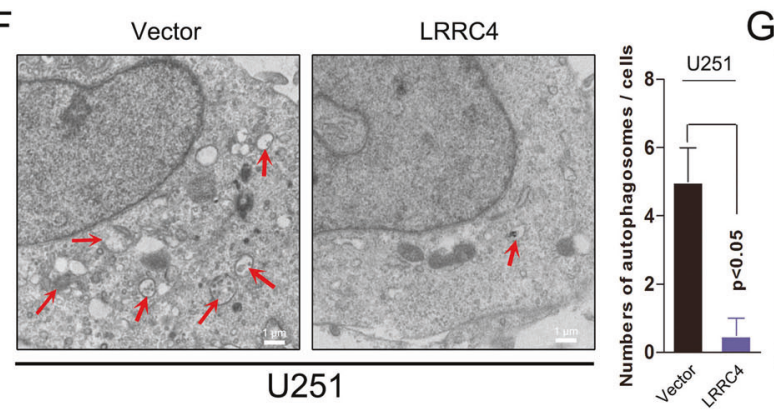

G
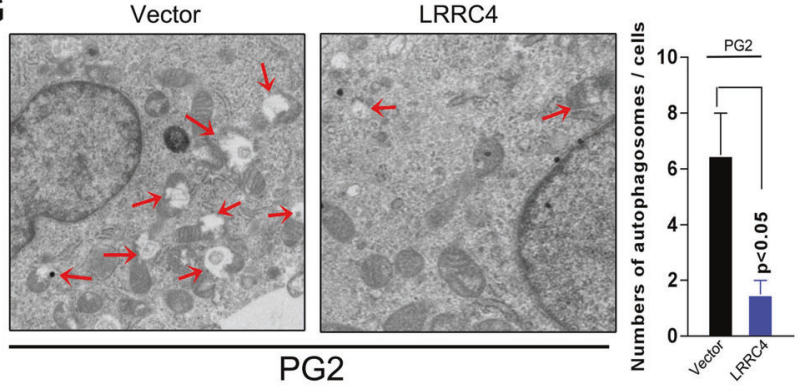

LRRC4 decreased the level of endogenous LC3II in U251 cells and PG2 cells (Fig. 2e). In addition, autophagosomes were evaluated. Transmission electron microscopy showed a decrease in the formation of autophagic vesicles in the LRRC4-overexpressing U251 and PG2 cells (Fig. 2f). Collectively, these data indicated that LRRC4 inhibits the autophagy pathway in GBM cells.

\section{LRRC4 $^{-l-}$ mice display relatively higher levels of autophagy than LRRC4 $^{+/+}$mice}

To further validate the effect of LRRC4 on autophagy in vivo, we generated knockout $(\mathrm{KO})$ mice that lacked exon 1 of the murine LRRC4 gene. Immunohistochemistry was used to analyse the expression of LRRC4, BECN1 and LC3B in E14.5 mouse embryos. Compared with $\mathrm{LRRC}^{+/+}$ 
Fig. 2 LRRC4 inhibits GBM cell autophagy activation. a The stably expressed LRRC4 decreased the expression of LC3B in U251 and PG2 cells. Knockdown LRRC4 restored the expression of LC3B in cells that stably expressed LRRC4. Cells were lysed and protein was extracted. LRRC4, LC3B and GAPDH were evaluated by western blotting. Vector: control plasmid, C4: LRRC4 plasmid. siC4-1 and siC4-2 are siRNAs that targeted LRRC4. Numbers represent the relative ratio of LC3II and GAPDH. b U251 cells were transfected with increasing concentrations of LRRC4 plasmid. Cells were lysed and protein was extracted. LRRC4, LC3B, P62 and GAPDH were evaluated by western blotting. LRRC4: LRRC4 plasmid. Numbers represent the relative ratio of LC3II and GAPDH, P62 and GAPDH. c U251 cells were transfected with LRRC4 or control plasmid and cells were cultured in normal and starvation conditions. LRRC4, LC3B, P62 and GAPDH were evaluated by western blotting. Vector: control plasmid, C4: LRRC4 plasmid. Numbers represent the relative ratio of LC3II, P62 and GAPDH. d U251 cells (upper) and PG2 cells (bottom) that stably expressed mRFP-EGFP-LC3 fusion protein were transfected with LRRC4 plasmid. Confocal microscopic analysis is shown. Scale bar, $5 \mu \mathrm{m}$. Vector: control plasmid, C4: LRRC4 plasmid. Note: PG2 cells were primary cultured glioblastoma cells. e U251 cells (upper) and PG2 cells (bottom) were transfected with LRRC4 plasmid. Cells were immunostained with an LC3B antibody. Fluorescence microscope analysis is shown. Scale bar, $20 \mu \mathrm{m}$. Vector: control plasmid, C4: LRRC4 plasmid. f, $\mathbf{g}$ Autophagosomes were observed by transmission electron microscopy in U251 cells and PG2 cells that were transfected with LRRC4 plasmid or control vector. Scale bar, $1 \mu \mathrm{m}$. Vector: control plasmid, LRRC4: LRRC4 plasmid.

wild-type mice, the expression of BECN1 and LC3B was increased in LRRC4 ${ }^{-1-}$ mice (Fig. 3a). Because LRRC4 is primarily expressed in the adult mouse nervous system, we next evaluated the expression of BECN1 and LC3B in the $\mathrm{LRRC}^{-1-}$ adult mouse nervous system. As shown in Fig. $3 \mathrm{a}$, $\mathrm{c}$ in both the mouse brain and spinal cord, the expression of BECN1 and LC3B was higher in $\mathrm{LRRC} 4^{-/-}$ mice than in $\mathrm{LRRC}^{+/+}$mice. These data demonstrated that LRRC4 plays an important role in regulating autophagy signals under physiological conditions in mice. The above results also demonstrated that LRRC4 inhibits cell autophagy both in physiological and pathological conditions.

\section{LRRC4 restores GBM cell sensitivity to TMZ via the autophagy pathway}

It is well known that autophagy activation contribute to drug resistance. Thus, we hypothesized that LRRC4 could decrease GBM chemoresistance by inhibiting autophagy. To initially determine whether LRRC4 decreases GBM chemoresistance, LRRC4 stably expressing U251 and PG2 cells were treated with different TMZ concentrations, with doses ranging between 0 and $700 \mu \mathrm{M}$. We observed that the half inhibitory concentration (IC50) of TMZ in U251 cells with LRRC4 expression was $\sim 122.3 \mu \mathrm{M}$; however, in control U251 cells, the IC50 was $\sim 221.5 \mu \mathrm{M}$ (Fig. 4a). The IC50 of TMZ was $\sim 138.7 \mu \mathrm{M}$ in PG2 cells with LRRC4 expression, whereas in the control PG2 cells, the IC50 was $\sim 181.2 \mu \mathrm{M}$ (Fig. $4 \mathrm{~b}$ ). These results showed that TMZ sensitivity was restored upon LRRC4 was expression in GBM cells. To further examine this hypothesis, cell apoptosis was examined by flow cytometry. As expected, TMZ induced U251 cell and PG2 cell apoptosis, and apoptosis increased in LRRC4-expressing U251 cells and PG2 cells compared with control cells (Fig. 4c, d). To address whether LRRC4 modulates the TMZ sensitivity of GBM cells via the autophagy pathway, we treated these cells with TMZ in the presence of chloroquine (CQ) or siRNA-mediated ATG5 and ATG7 gene knockdown. We found that the LRRC4-regulated TMZ-sensitivity effect was increased by CQ treatment (Fig. 4e, f) or knockdown of ATG5 or ATG7 in U251 cells and PG2 cells (Fig. S2). Moreover, in the presence of CQ, western blotting also showed that TMZ induced the cleavage of caspase 3, caspase 7 and PARP, and these effects were enhanced by LRRC4 expression in U251 cells and PG2 cells (Fig. 4g, h). Thus, these data suggest that LRRC4 restores TMZ-sensitivity in GBM by inhibiting autophagy and promoting apoptosis.

\section{LRRC4 combined with TMZ treatment prolonged mouse survival}

The profound proapoptotic effects of the combination of LRRC4 expression and TMZ treatment in vitro suggest that targeting autophagic signalling may be a rational strategy for the treatment of GBM. To explore the feasibility of this therapeutic strategy, we tested the efficiency in tumour xenografts in vivo. U251-Luci-GFP-Vector or U251-LuciGFP-LRRC4 cells were implanted into the brain of nude mice, and 14 days later, mice received intraperitoneal injections of TMZ $(40 \mathrm{mg} / \mathrm{kg} / \mathrm{d})$ or saline once a day for 7 days (Fig. 5a). Imaging using an IVIS Lumina III In Vivo Imaging System showed no significant difference in the fluorescence signal in mice engrafted with U251-Luci-GFPLRRC4 cells in comparison with that of animals implanted with U251-Luci-GFP-Vector cells (Fig. 5b, $p=0.0529$ ). Nonetheless, when treated with TMZ, mice engrafted with U251-Luci-GFP-LRRC4 cells showed a significant decrease in fluorescence signal compared with that of mice implanted with U251-Luci-GFP-Vector cells (Fig. 5b). The relative fluorescence quantification is shown in Fig. 5c. Moreover, survival analysis showed that when mice that were intracranially engrafted with U251-Luci-GFP-LRRC4 cells were treated with TMZ, survival was longer than in animals implanted with U251-Luci-GFP-Vector cells (Fig. 5d) $(p<0.05)$. Immunofluorescence was used to confirm whether the decreased tumour growth observed in mice with LRRC4 overexpression combined with TMZ treatment was associated with an autophagy defect. Immunofluorescence staining in mouse brain sections 
A
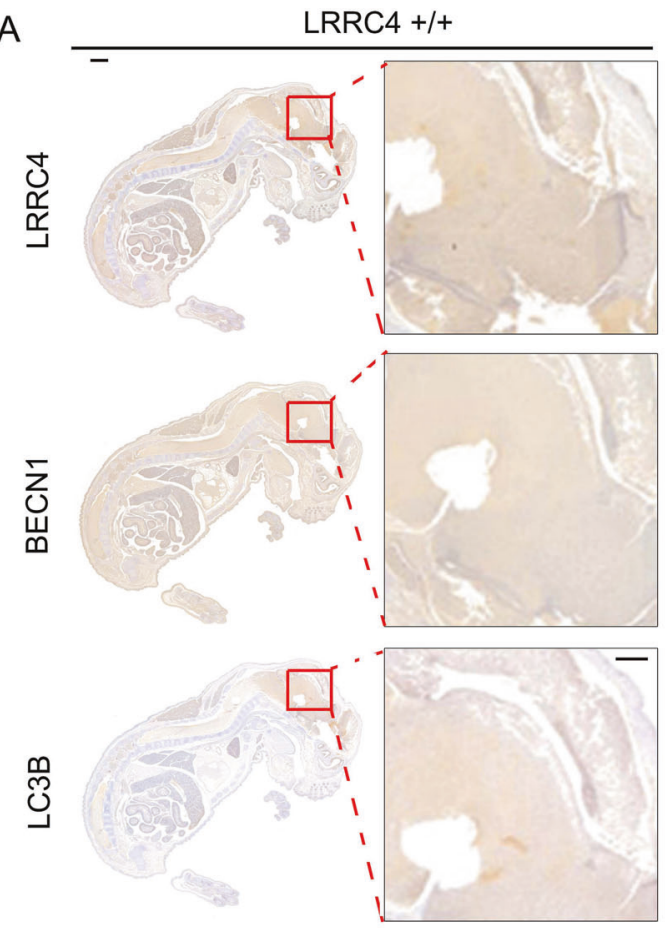

B

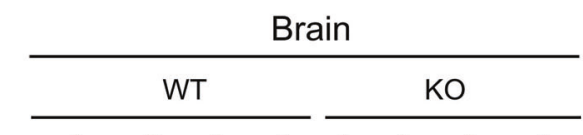

No.

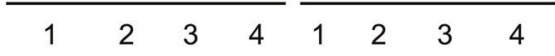

LRRC4

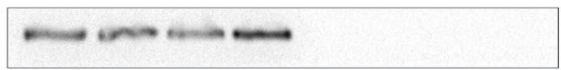

BECN1

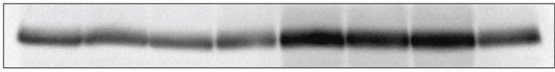

LC3B

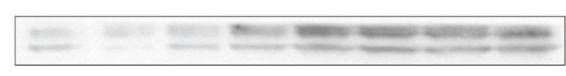

GAPDH

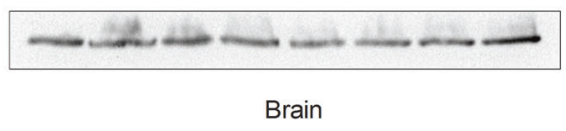

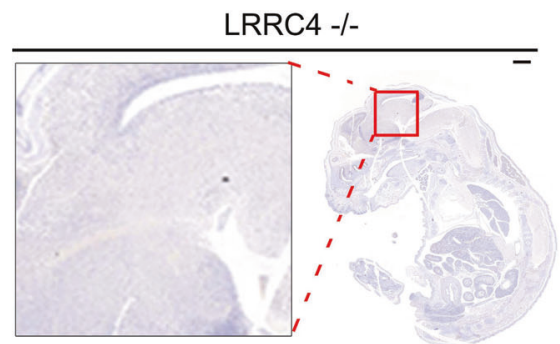
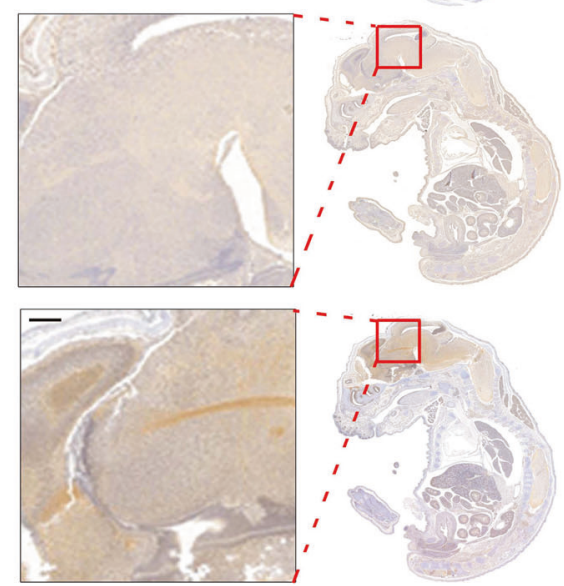

C
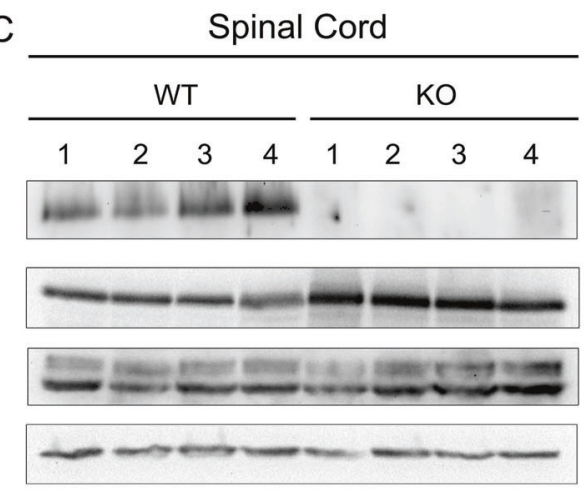

Spinal Cord

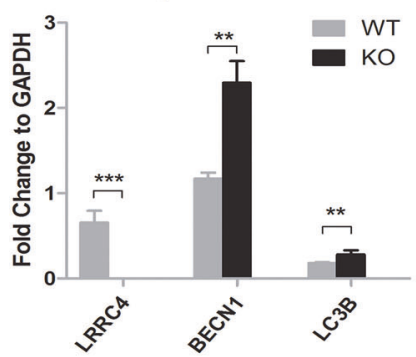

scale bar, $100 \mu \mathrm{m}$. Representative western blotting analysis of LRRC4, BECN1, LC3B and GAPDH in adult mouse brain (b) and spinal cords (c) from LRRC4 WT and KO mice. Statistical analysis is shown at the bottom.

\section{LRRC4 binds to DEPTOR via the C-terminal PDZ binding domain}

To investigate the mechanism by which LRRC4 inhibited cell autophagy, we used co-immunoprecipitation (CoIP) 
A

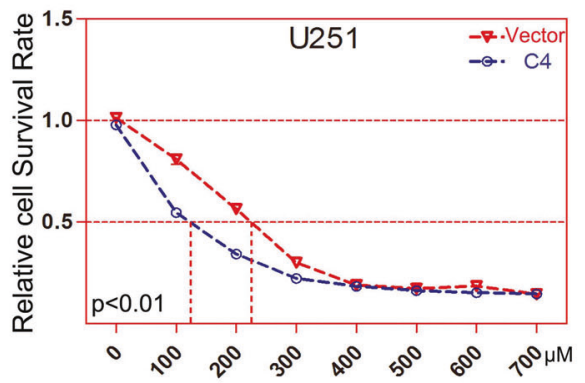

C
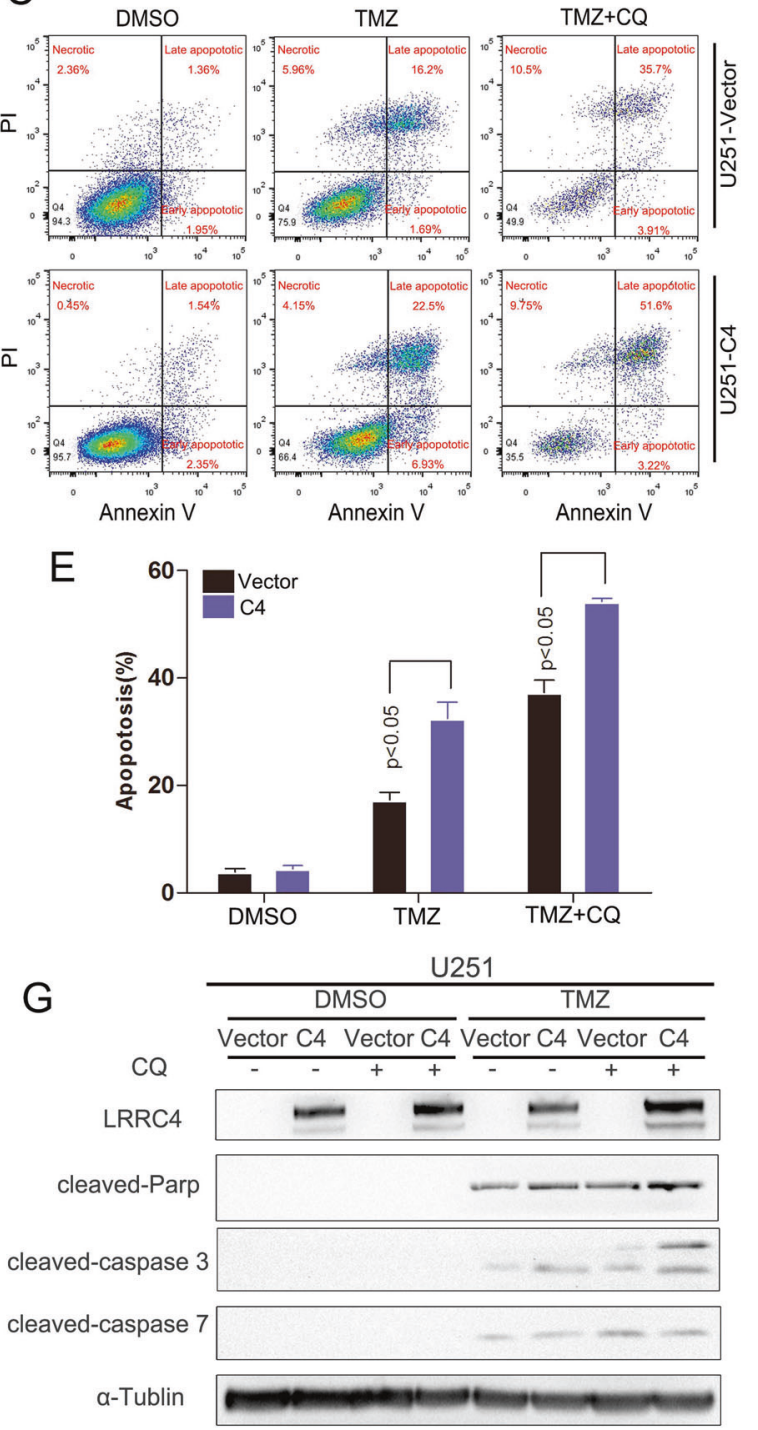

Fig. 4 LRRC4 restores GBM cell sensitivity to TMZ. a, b U251 cells (left) and PG2 cells (right) that stably expressed control vector or LRRC4 (C4) were treated with different concentrations of TMZ. Cell viability was evaluated by CCK-8. c, d Apoptosis was detected by flow cytometry in U251 cells (left) and PG2 cells (right) that stably expressed control vector or LRRC4 (C4). The cells were treated with CQ (chloroquine) and TMZ (temozolomide). The images
B

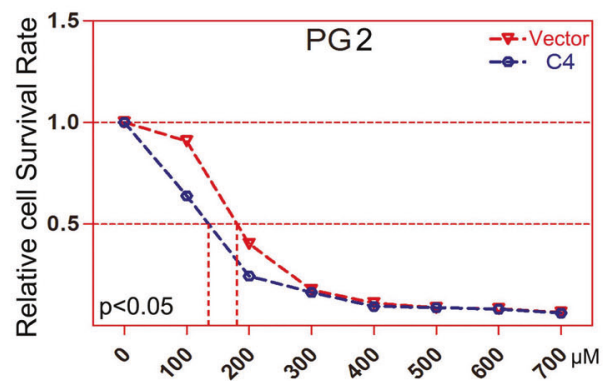

D

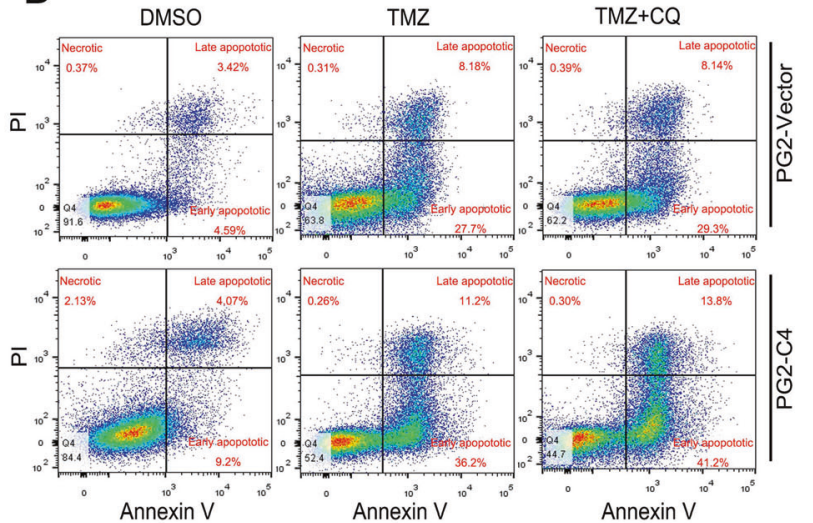

$\mathrm{F}$

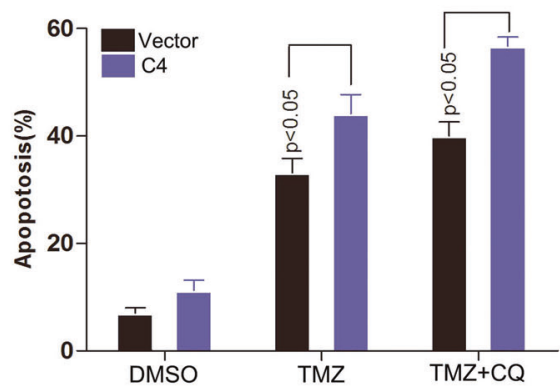

$\mathrm{H}$
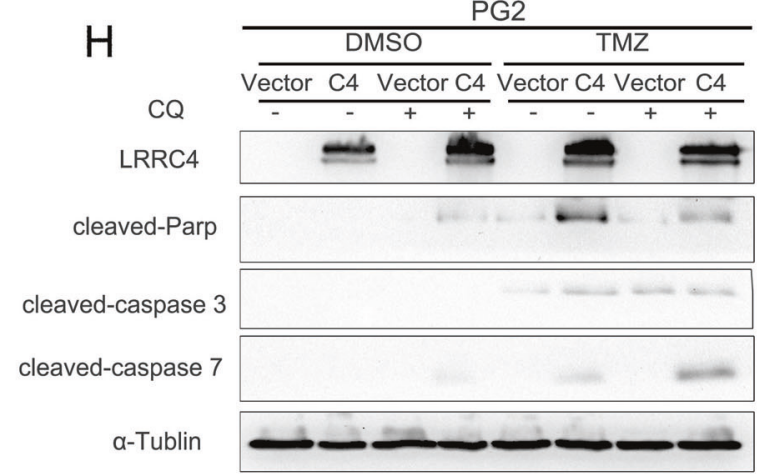

are representative of three independent experiments. e, f Statistical analysis was performed to calculate the apoptosis rate in $\mathbf{c}$ and $\mathbf{d} . \mathbf{g}, \mathbf{h}$ Cleaved caspase 3,7 and parp expression was detected by western blotting in U251 cells (left) and PG2 cells (right). The cells were treated with CQ and TMZ. Vector: control plasmid, C4: LRRC4 plasmid. 
A Experimental design
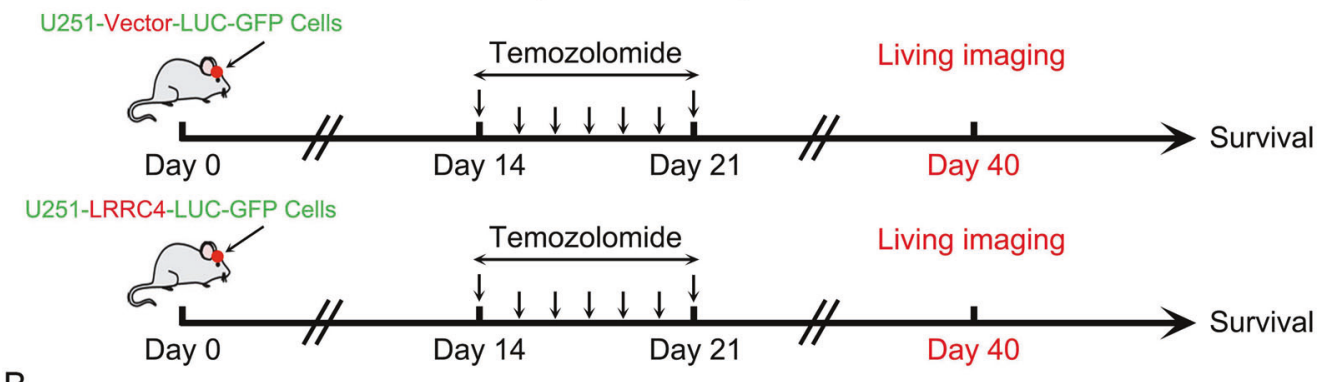

B
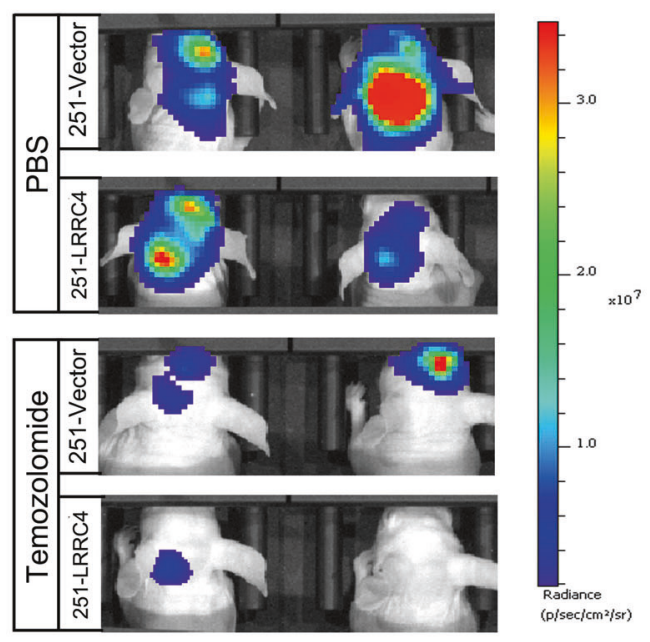

E
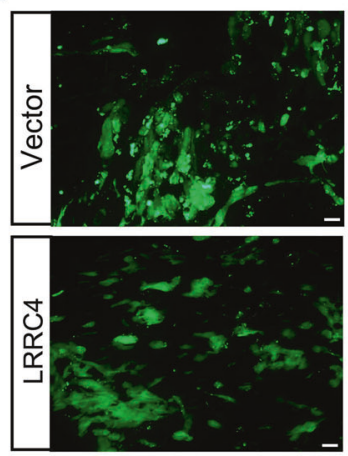

U251-GFP/LC3B/DAPI
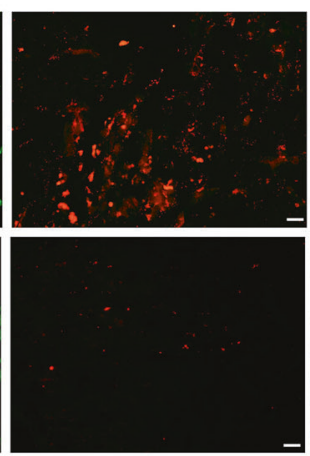

Fig. 5 LRRC4 combined with TMZ treatment prolonged mouse survival. a Experimental design schematic showing the in vivo mouse model. b Representative bioluminescence images of mice intracranially injected with luciferase-labelled U251 cells that stably expressed vector or LRRC4 in the presence or absence TMZ treatment $(n=6$ mice per group). c Quantitation of the fluorescence intensity of a representative image $\left(n=6\right.$ mice per group). ${ }^{* *} p<0.01$; d

combined with mass spectrometry to identify its interaction partners, and DEPTOR (DEP domain containing MTOR interacting protein) was identified as a potential LRRC4 interaction partner (Fig. S3). DEPTOR interacts with both mTORC1 and mTORC2, and inhibits the activity of both mTORC1 and mTORC2 [29]. It is recognized that mTOR regulate autophagy through direct phosphorylation of

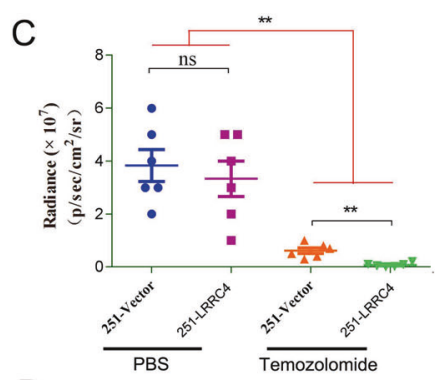

D
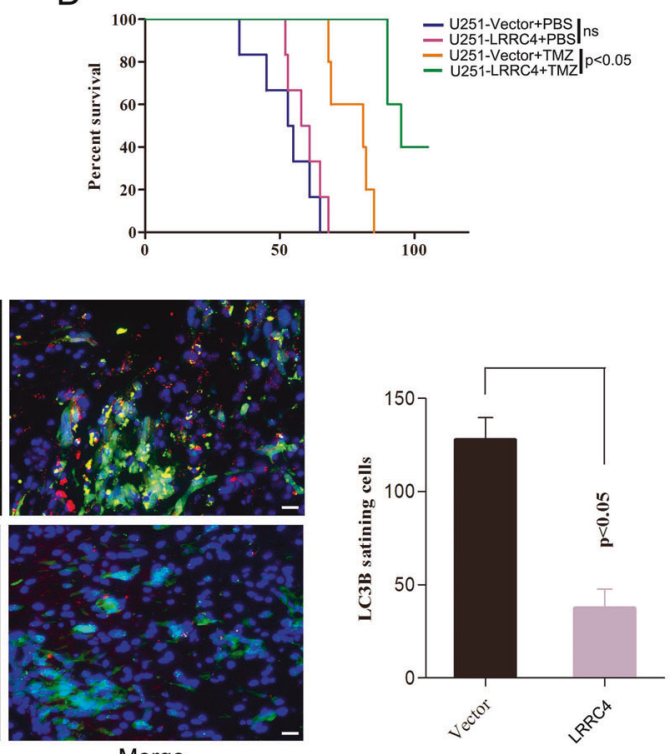

Kaplan-Meier survival curves of mice injected with U251 cells that stably expressed vector or LRRC4 in the presence or absence TMZ treatment ( $n=6$ mice per group). e Mouse brain sections stained for LC3B after intracranial transplantation of U251 cells that stably expressed vector or LRRC4. The right panel shows the quantitation of LC3B-positive cells.

Ulk1 [30]. So we then decided to concentrate on the DEPTOR.

To test whether LRRC4 interacted with endogenous DEPTOR, a FLAG-tagged LRRC4 protein expression vector was transfected into HEK293 cells. The endogenous DEPTOR was co-immunoprecipitated with LRRC4 from the cell extract (Fig. 6a). Consistently, endogenous 
A

D

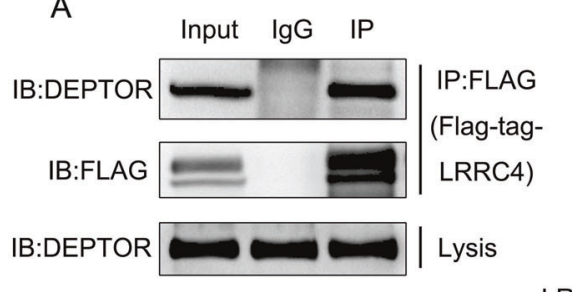

Input

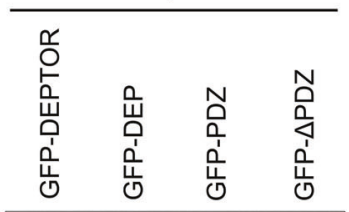

LRRC4

B

Input IgG IP

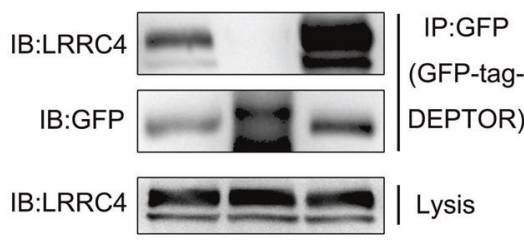

C

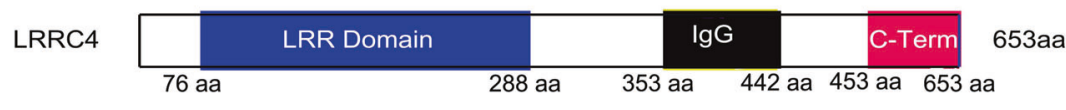

409aa

E
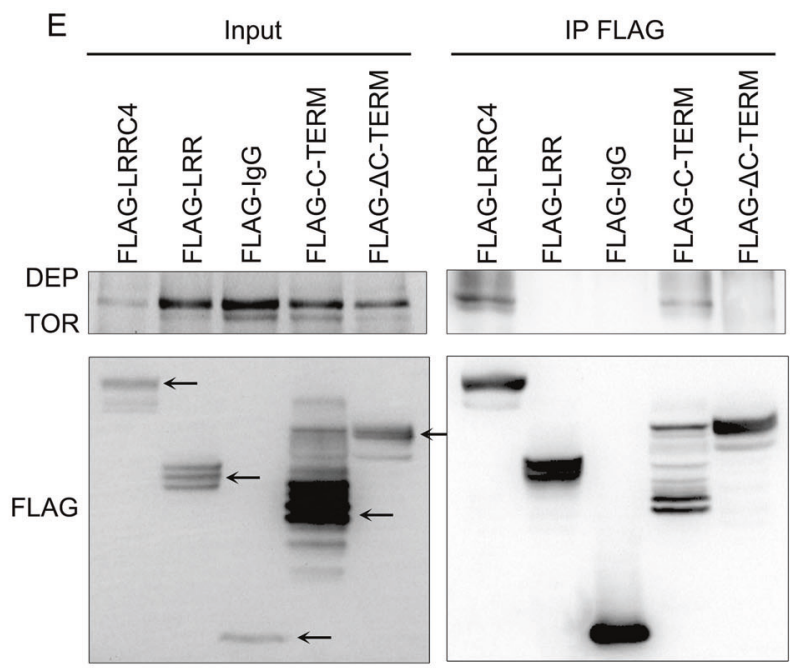

$\mathrm{F}$

GST-Pull Down

Fig. 6 LRRC4 interacted with DEPTOR. a U251 cells were transfected with FLAG-LRRC4. Co-immunoprecipitation showed the interaction between LRRC4 and endogenous DEPTOR in U251 cells. b U251 cells were co-transfected with EGFP-C1-DEPTOR and pCDNA-3.1-LRRC4 plasmid. Co-immunoprecipitation showed the interaction between LRRC4 and DEPTOR in U251 cells. c Schematic diagrams of LRRC4 (upper)/DEPTOR (bottom) protein domains. d HEK293 cells were co-transfected with different GFP-tagged domains

LRRC4 could also co-immunoprecipitate with GFPtagged DEPTOR from the cell extract (Fig. 6b). To identify the interacting regions, we subsequently constructed plasmids containing different domains of LRRC4 and DEPTOR to perform CoIP assays. PDZ(330-407), containing the PDZ domain of DEPTOR, but not DEP (1329), containing the DEP domain of DEPTOR, interacted with LRRC4, and deletion of the PDZ domain abolished of DEPTOR and the pCDNA3.1-LRRC4 plasmid. Coimmunoprecipitation showed the interaction between LRRC4 and the PDZ domain of DEPTOR. e HEK293 cells were co-transfected with different FLAG-tagged domains of LRRC4 and pCDNA3.1DEPTOR. Co-immunoprecipitation showed the interaction between DEPTOR and the C-terminal domain of LRRC4. f GST pull-down assays showed that the PDZ domain of DEPTOR pulled down LRRC4.

the interaction between LRRC4 and DEPTOR (Fig. 6d). LRRC4 (443-653), containing the PDZ binding domain, but not other LRRC4 regions interacted with DEPTOR, and deletion of the C-terminal PDZ binding domain also abolished the interaction between LRRC4 and DEPTOR (Fig. 6e). In order to determine direct protein-protein interaction between DEPTOR and LRRC4, we subsequently performed a GST pull-down assay with LRRC4 
and GST-fused DEPTOR full-length or different domains of DEPTOR (DEP: DEP domain, PDZ: PDZ domain or $\triangle$ PDZ: detection of PDZ domain in DEPTOR protein) as shown in Fig. 6c. The results revealed that the LRRC4 was pulled down by either the GST-fused full-length DEPTOR or GST-fused PDZ domain of DEPTOR, whereas the GST-fused DEP or $\triangle \mathrm{PDZ}$ was not able to pull-down LRRC4 (Fig. 6f). Collectively, these data demonstrated that LRRC4 binds to DEPTOR directly through the C-terminal PDZ binding domain of LRRC4 and the PDZ domain of DEPTOR.

\section{LRRC4 promoted the degradation of the DEPTOR protein and re-expression of DEPTOR restored autophagy activation}

DEPTOR is an unstable protein and can be degraded under growth factor stimulation [29]. Previous studies also reported that DEPTOR was degraded by $\mathrm{SCF}^{\beta \mathrm{TrCP}}$ [30]. Next, we wanted to determine whether the interaction between LRRC4 and DEPTOR could alter the expression of DEPTOR. We found that DEPTOR expression was decreased in $\mathrm{U} 251$ and PG2 cells that were transfected with an LRRC4 plasmid while not in PG1 cells (Fig. 7a). In line with that the overexpression of LRRC4 in PG1 has no effect on autophagy inhibition. It suggested that LRRC4 inhibited autophagy via downregulating DEPTOR. We next examined whether LRRC4 affected the stability of DEPTOR using a cycloheximide (CHX)-chase assay. The half-life of DEPTOR was decreased by overexpression of LRRC4 in U251 cells (Fig. 7b). In addition, we also examined the effect of overexpressing LRRC4 on DEPTOR ubiquitin modifications, and the ubiquitin modifications of DEPTOR were also increased by transfected LRRC4 (Fig. 7c).

To further demonstrate the effects of regulation by LRRC4 on DEPTOR expression and autophagy, U251 and PG2 cells were transiently transfected with LRRC4 plasmid. The overexpression of LRRC4 could induce mTOR activation, as shown by increased expression of phosphoMTOR and phospho-S6K (Fig. 7d), which was consistent with the precious conclusion that the cell autophagy level could be inhibited by MTOR pathway activation. Furthermore, when GBM cells treated with pharmacological MTOR inhibitors (Rapamycin), the inhibition effect of LRRC4 on autophagy was broke, as the difference of p62 and LC3B protein level between vector and LRRC4expressing group had no significant changes (Fig. 7e). Moreover, re-expression of DEPTOR rescued cell autophagy in LRRC4 stable expression U251 cells, as Fig. 7f shows increased protein levels of LC3B. Similarly, reexpression of DEPTOR could restore the formation of autophagic vesicles in LRRC4-expressing U251 cells
(Fig. $7 \mathrm{~g}$ ). Together, these results demonstrate that LRRC4 inhibits cell autophagy by promoting DEPTOR degradation and that re-expression of DEPTOR blocks this suppressive effect.

\section{The levels of LRRC4, DEPTOR and LC3B are clinically relevant for GBM}

To investigate the clinical significance of LRRC4, DEPTOR and LC3B in GBM, we detected the protein expression of LRRC4, DEPTOR and LC3B by immunohistochemistry in GBM tissues. We found that high expression of LRRC4 was more likely to be detected in patients with low expression of DEPTOR and LC3B. In contrast, low expression of LRRC4 was more likely detected in patients with high expression of DEPTOR and LC3B (Fig. 8a). Furthermore, we examined the LRRC4, LC3B and DEPTOR protein expression in total protein extraction of GBM specimens. As showed in Fig. 8b, DEPTOR was expressed in most GBM tissues, while in LRRC4 expressed tissues (T1, T3, T6 and T9), the expression of DEPTOR and LC3B protein was relative low compared with no-LRRC4 expression tissues (Fig. 8c).

We also studied the relationship of LRRC4 and DEPTOR in Cohort 1 and Cohort 2, as mentioned in Fig. 1. Similarly, LRRC4 was also negatively correlated with DEPTOR in Cohort 1 and Cohort 2 (Fig. 8d). As drug resistance contribute to tumour recurrence, we also evaluated the expression of LRRC4 mRNA in GBM and recurrent GBM patients by using the Oncomine Murat brain dataset. As shown in Fig. 8e, reduced LRRC4 mRNA levels were found in recurrent GBM samples compared with GBM samples without recurrence, suggesting that the expression levels of LRRC4 may be negatively correlated with drug resistance. Altogether, we conclude that LRRC4 may act in GBM via DEPTOR, causing MTOR pathway activation, which subsequently results in autophagy inhibition, and consequently promotes TMZ chemosensitivity in GBM patients (Fig. 8f).

\section{Discussions}

The LRRC4 gene was first identified on human chromosome 7q31-32 by our group [19], and our reports confirmed that LRRC4 is a tumour suppressor gene for glioma [27, 28]. Although the function of LRRC4 has been reported in our previous work, it is unknown whether LRRC4 may mediate autophagy in glioma. We found that expression of LRRC4 inhibited autophagic flux and autophagosome synthesis in GBM cells. Accordingly, LRRC4 decreased the expression of the autophagy-related proteins LC3B in GBM cells, suggesting that LRRC4 

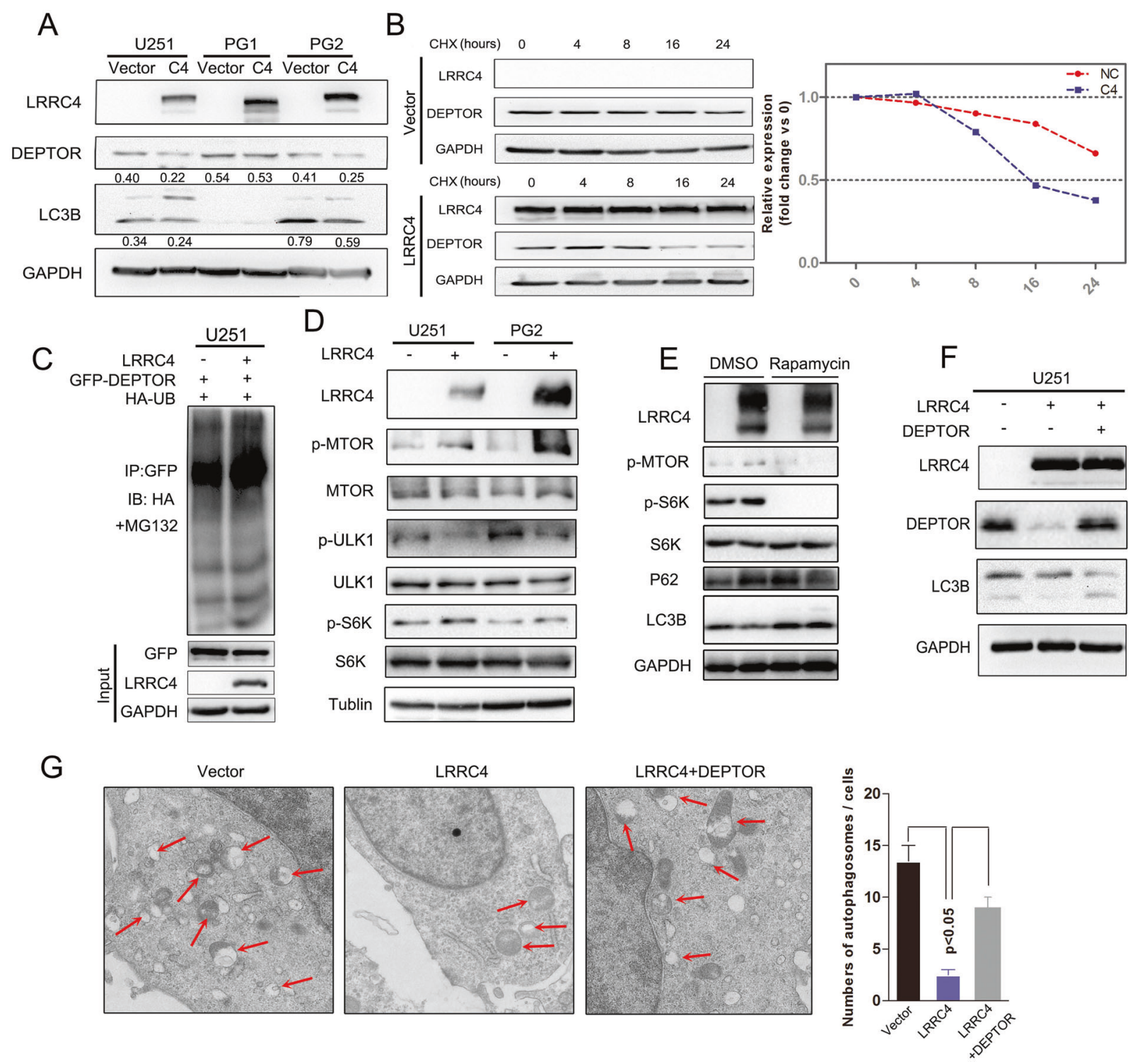

Fig. 7 LRRC4 promoted the degradation of DEPTOR protein, and re-expression of DEPTOR restored autophagy activation. a Representative western blotting analysis of LRRC4, DEPTOR, LC3B and GAPDH in U251, PG1 and PG2 cells with or without transfection with LRRC4. Numbers represent the relative ratio of DEPTOR/LC3B and GAPDH. b Western blotting analysis was used to measure of the half-life of DEPTOR after treatment with cycloheximide in U251 cells with (down) or without (upper) LRRC4 transfection. Cells were lysed and protein was extracted at $0,4,8,16,24 \mathrm{~h}$ after cycloheximide treatment. c DEPTOR ubiquitination was assessed by an anti-GFP antibody in the presence of MG132 when GFP-DEPTOR, HA-ubiquitin, and LRRC4 or vector were co-transfected into U251 cells. d

prevents autophagy pathway activation. We also examined the relationship of LRRC4 and autophagy in LRRC4 gene $\mathrm{KO}$ mice and found that the lrrc $4^{-1-}$ mice display relatively high levels of autophagy-related proteins compared with those of WT mice in mouse brain and spinal cord. Previous studies have shown that LRRC4 regulates auditory responses [31], excitatory synapse development, synapse
Representative western blotting analysis of LRRC4, p-MTOR, MTOR, p-ULK1, ULK1, p-S6K, S6K and Tubulin in U251 and PG2 cells with or without transfection with LRRC4. e Representative western blotting analysis of LRRC4, p-MTOR, p-S6K, S6K, P62, LC3B and GAPDH in U251 cells, U251 cells with or without LRRC4 transfection were treated with dimethylsulfoxide (DMSO) or Rapamycin. f Representative western blotting analysis of LRRC4, DEPTOR, LC3B and GAPDH in U251 cells, U251 cells with stable expression LRRC4 and LRRC4-expressing U251 cells with ectopic expression of DEPTOR. $\mathbf{g}$ Autophagosomes were observed by transmission electron microscopy in U251 cells, U251 cells with stable expression LRRC4 and LRRC4expressing U251 cells with ectopic expression of DEPTOR.

maintenance and restoration in the retina [32] and autisticlike behaviours that are responsive to NMDAR modulation [33] by using lrrc4 KO mice. Our unpublished results showed that lrrc $4^{-1-}$ mice display a more serious phenotype than WT mice in the EAE model, and re-expressing LRRC4 in $1 \mathrm{rrc}^{-1-}$ mice could partially rescue the phenotype. Autophagy dysfunction in neurodegenerative disorders has 
A

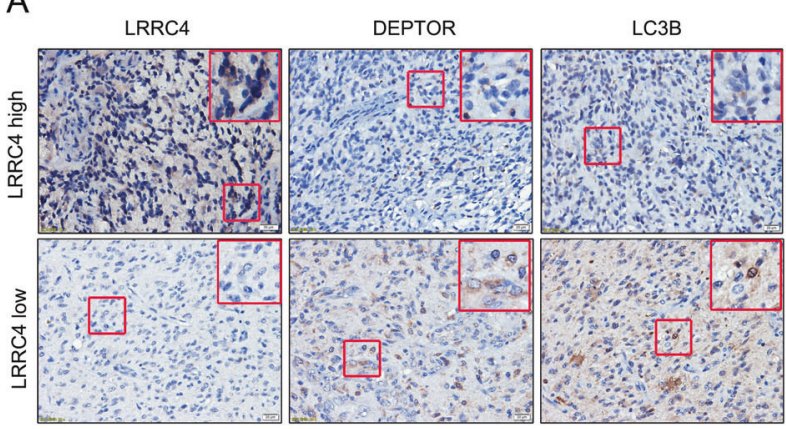

B

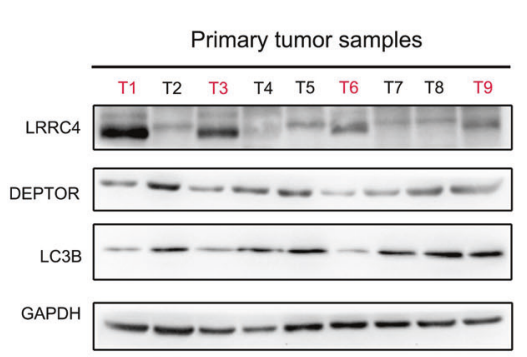

C

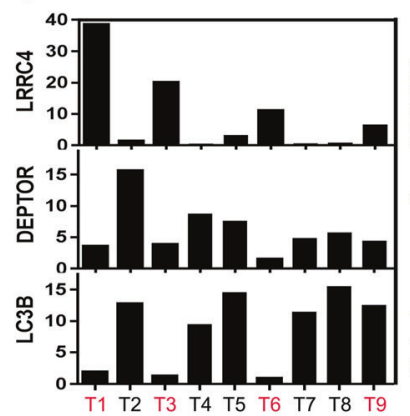

D

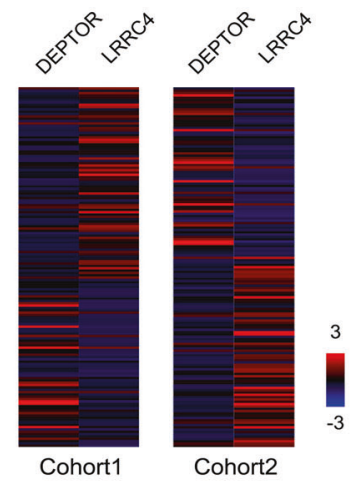

E

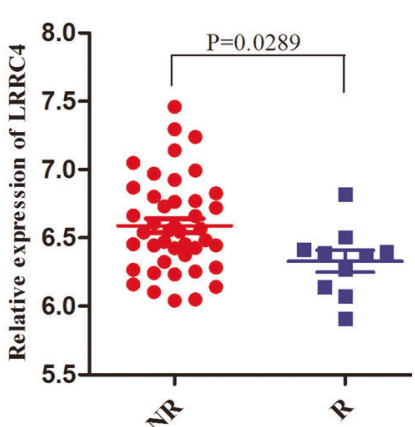

$\mathrm{F}$

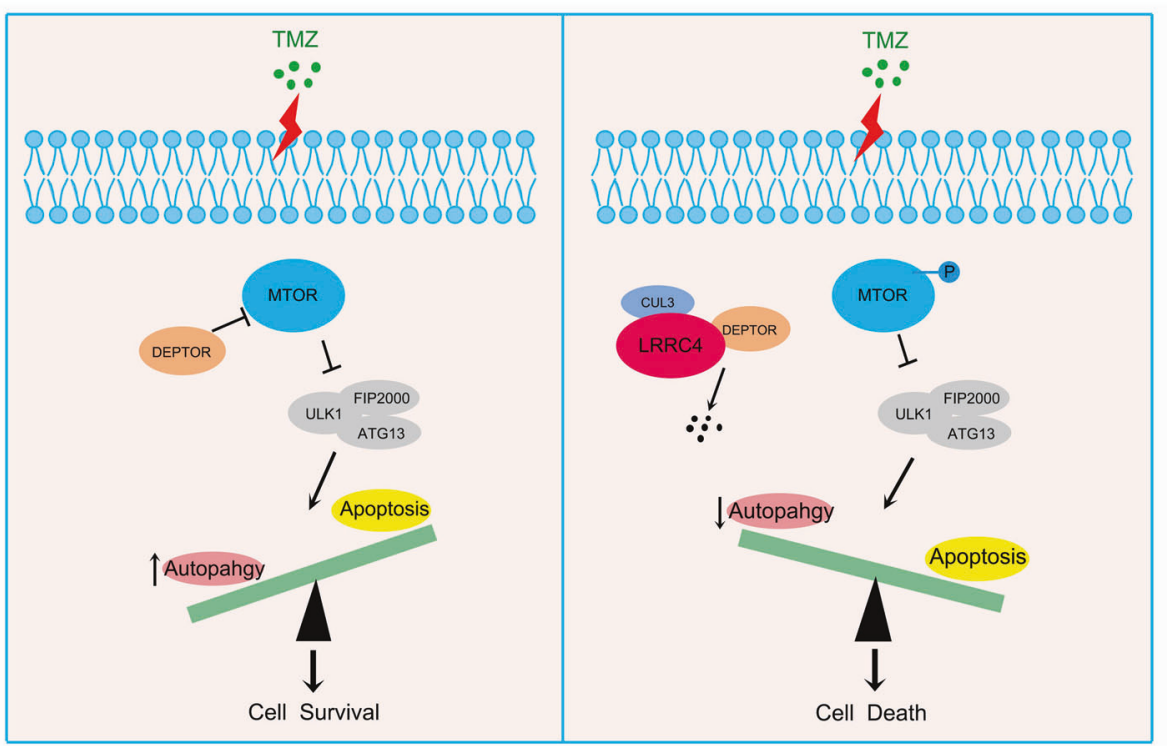

Fig. 8 The Levels of LRRC4, DEPTOR and LC3B are clinically relevant for GBM. a Representative immunohistochemistry staining of LRRC4, DEPTOR and LC3B proteins in GBM tissues. (LRRC4 high GBM tissues =5, LRRC4 low GBM tissues =27). b Representative western blotting analysis of LRRC4, DEPTOR and LC3B proteins in primary GBM tissues. LRRC4 is negatively correlation with DEPTOR and LC3B expression. T tumour. $\mathbf{c}$ The quantitative analysis of LRRC4, DEPTOR and LC3B proteins expression in each primary GBM tissues (b). d The correlation analysis of LRRC4 and DEPTOR in Cohort 1 (Glioblastoma, TCGA, Cell 2013, left) and Cohort 2 (Glioblastoma Multiforme, TCGA, PanCancer Atlas, right) of GBM samples from TCGA database. e Statistical analysis of the expression of LRRC4 between recurrent and non-recurrent patients from the Oncomine Murat brain dataset. f Schematic diagram of the relationship among LRRC4, autophagy and TMZ chemosensitivity: when GBM cells without LRRC4 expression are attacked by TMZ treatment, DEPTOR directly binds and inhibits the phosphorylation of MTOR, thereby trigger protective autophagy to permit GBM cell survival. But, when GBM cells with LRRC4 expression are attacked by TMZ treatment, LRRC4 directly binds and promotes the degradation of DEPTOR. Degradation of DEPTOR leads to activation of MTOR, thereby inhibits protective autophagy, and finally induces apoptotic cell death. 
been widely reported [34, 35]. Our study reveals that LRRC4 regulates autophagy in the mouse nervous system. This may explain why LRRC4 dysfunction contributes to neurological function disorders in a mouse model.

TMZ, an FDA-approved chemotherapy drug, has been widely used to treat glioma [36]. Although glioma patients often initially respond to surgical resection and chemotherapy, relapse of drug-resistant cancer usually occurs, and treatment is usually ineffective [37]. Unfortunately, due to the existence of the blood-brain barrier, potentially powerful anticancer drugs and novel immune checkpoint therapy are ineffective for GBM [38]. TMZ remains a firstline therapy for patients with GBM. Thus, understanding the mechanisms of TMZ resistance in GBM or exploring prognostic markers that predict TMZ chemosensitivityis essential to optimize current therapeutic strategies. It has been reported that chemotherapy can induce autophagy activation in tumour cells, and some articles have also discussed a strategy that targets autophagy to sensitive glioma to TMZ treatment [39-41]. Our study found that TMZ treatment induced the expression of autophagy-related proteins BECN1 and LC3B (data not shown). Hence, we hypothesized that LRRC4 expression could promote the sensitivity of GBM to TMZ treatment. We confirmed that LRRC4 induced GBM cell apoptosis when treated with TMZ, and the combination of biochemical autophagy inhibition (CQ) with LRRC4 expression significantly enhanced the cell apoptosis rate. Thus, we conclude that autophagy contributes to LRRC4-mediated GBM responses to TMZ regimens. These results support the phenomenon that GBM patients with low expression of LRRC4 experience poor outcomes and low TMZ chemosensitivity.

We have described the mechanisms by which LRRC4 inhibits autophagy pathway activation. DEPTOR was found to interact with LRRC4 by MS analysis. DEPTOR is a naturally occurring inhibitor of mTOR that directly binds to both mTORC1 and mTORC2 [29]. DEPTOR is subject to proteasome-dependent degradation [30], and the degradation of DEPTOR contributes to mTOR activation, thus inhibiting the cell autophagy pathway [42]. Our data showed that LRRC4 induces the degradation of DEPTOR by directly interacting with DEPTOR. We also confirmed that overexpression of LRRC4 induced phosphorylation of mTOR and S6K1, which was accompanied by decreased expression of the autophagyrelated proteins LC3B. This result supports the conclusion that LRRC4 inhibits GBM cell autophagy via the degradation of DEPTOR. DEPTOR acts as a tumour suppressor by blocking mTORC1 and mTORC2, inhibiting cell proliferation. However, studies have also demonstrated that DEPTOR is overexpressed in many tumours, including breast, prostate and lung cancers [43-45], indicating that DEPTOR also acts as an oncogene during tumour growth. DEPTOR overexpression is able to inhibit mTORC1, leading to an apparent increase in
mTORC2 signalling, inducing Akt phosphorylation at S437 and T308 residues [46]. Efeyan found that DEPTOR could relieve the feedback inhibition from S6K1 to PI3K, thus activating AKT [47]. Wang also reported that DEPTOR was a novel target of Wnt/b-Catenin/c-Myc and contributed to colorectal cancer cell growth [48]. This may explain why LRRC4 expression leads to mTOR activation but does not contribute to cell proliferation.

In conclusion, our results demonstrate that LRRC4, which is frequently deregulated in glioma, directly binds to DEPTOR and induces its degradation to activate mTOR, thereby inhibiting cell autophagy. Moreover, autophagy inhibition increased the treatment efficacy of TMZ in glioma, and LRRC4-expressing cells underwent increased apoptosis with TMZ treatment. Importantly, in clinical glioma samples, LRRC4 was also negatively associated with DEPTOR and LC3 expression. Combined LRRC4 expression and TMZ treatment could be an effective strategy for glioma therapy. Thus, the expression of LRRC4 is likely to have significant potential as a therapeutic marker and target for TMZ treatment in glioma patients.

\section{Materials and methods}

\section{Tissue samples}

Primary glioma samples and normal brain tissue were obtained from the Department of Neurosurgery at Xiangya Hospital in Hunan, China. All of the protocols were reviewed by the Joint Ethics Committee of the Central South University Health Authority and performed following national guidelines. Primary tumour and normal brain tissue were frozen in liquid nitrogen and stored until total proteins were extracted.

\section{Primary cell culture}

Primary cell (PG1 and PG2) culture protocols were described in our previous report [28].

\section{Cell culture and reagents}

U251, HEK293, PG1 and PG2 cells were maintained in DMEM supplemented with $10 \%$ foetal bovine serum and antibiotics. Cells were incubated at $37^{\circ} \mathrm{C}$ in a humidified atmosphere of $5 \% \mathrm{CO}_{2}$. U251 cells were authenticated to have originated from ATCC by short tandem repeat profiling. For Starvation induced autophagy, GBM cells were transfected with LRRC4 plasmid or control vector, after $42 \mathrm{~h}$, the cells were washed twice with D-hanks and were stimulated with EBSS (6h) as amino-acid starvation conditions. Cell extracts were immunoblotted with the indicated antibodies. Antibodies 
against GAPDH (60004-1-Ig), GST (66001-1-Ig), HA (510642-AP) and P62 (18420-1-AP) were purchased from Proteintech. Antibodies against LRRC4 (ab116697) were purchased from Abcam. FLAG (F1804) was purchased from Sigma-Aldrich. Antibodies against Beclin-1 (D40C5), LC3B (D11), mTOR (7C10), phospho-mTOR (Ser2448) (D9C2), DEPTOR/DEPDC6 (D9F5) and an apoptosis antibody sampler kit (\#9915) were purchased from Cell Signaling Technology.

\section{Immunoprecipitation and immunoblotting}

The protocols were described in our previous reports. Briefly, for immunoprecipitation, cells were lysed in IP buffer $(25 \mathrm{mM}$ Tris $\mathrm{pH} 7.5,150 \mathrm{mM} \mathrm{NaCl}, 1 \%$ Triton $\mathrm{X}-100$ and $1 \mathrm{mM}$ EDTA). Cell lysates were incubated with the indicated antibodies at $4{ }^{\circ} \mathrm{C}$ overnight. Protein A/G Magnetic Beads were washed twice with IP buffer and were then added to the reaction mixtures and incubated for $2 \mathrm{~h}$ at $4{ }^{\circ} \mathrm{C}$. After magnetic separation, the magnetic beads were washed four times with IP buffer and boiled for $10 \mathrm{~min}$ after the addition of $2 \times \mathrm{SDS}$ loading buffer. The immunoprecipitated proteins were analysed by SDS-PAGE. For immunoblotting, cells were lysed in RIPA buffer (100 mM Tris $\mathrm{pH} 7.4,150 \mathrm{mM} \mathrm{NaCl}, 5 \mathrm{mM}$ EDTA, $1 \%$ Triton X-100, $1 \%$ deoxycholate acid, $0.1 \%$ SDS, $2 \mathrm{mM}$ phenylmethylsulfonyl fluoride, $1 \mathrm{mM}$ sodium orthovanadate, $2 \mathrm{mM}$ DTT, $2 \mathrm{mM}$ leupeptin, $2 \mathrm{mM}$ pepstatin). Protein lysates were fractionated by SDS-polyacrylamide gel electrophoresis, transferred onto PVDF membranes (Merck Millipore, Germany), and then incubated with the indicated primary antibodies, washed and probed with HRP (Horseradish peroxidase)-conjugated secondary antibodies.

\section{Pull-down assay}

The protocol was described in our previous reports. Briefly, glutathione-S-transferase (GST) fusion proteins containing various regions of DEPTOR were expressed in BL21 (DE3) bacteria with a pGEX-4T-2 vector and were purified. Various FLAG-tagged sections of LRRC4 were transfected into HEK293 cells and were lysed in IP buffer. Then, the lysate was incubated for $4 \mathrm{~h}$ with GST-tagged proteins and glutathioneSepharose 4B beads. The beads were subsequently washed four times in IP buffer. Precipitates were separated by SDSPAGE and detected by western blot analysis.

\section{Immunofluorescence}

The cultured cells were plated on coverslips and transfected with the indicated plasmids. After $48 \mathrm{~h}$, the cells were washed and fixed in $4 \%$ paraformaldehyde at room temperature for $30 \mathrm{~min}$. The cells were then washed two times with $0.1 \%$ PBS-T. For permeabilization, cells were incubated with $0.25 \%$ Triton X-100 in PBS for 15 min and washed two times with $0.1 \%$ PBS-T. Cells were incubated in blocking solution (normal goat serum) for $30 \mathrm{~min}$ to block nonspecific binding of the antibody and were incubated in primary antibodies diluted in PBS. After four washes with $0.1 \%$ PBS-T, cells were incubated in secondary antibodies for $1 \mathrm{~h}$ at room temperature. Coverslips were mounted and imaged by fluorescence microscopy.

\section{Xenograft tumour model}

All animal experiments were approved by the Animal Care and Use Committee of Central South University. U251-GFPLuci-Vector or U251-GFP-Luci-LRRC4 cells were collected, resuspended at $5 \times 10^{5}$ cells in $5 \mu$ of serum-free medium per animal, and then stereotactically injected into the striatum $(1.0 \mathrm{~mm}$ anterior and $1.0 \mathrm{~mm}$ lateral from the Bregma suture and $3.0 \mathrm{~mm}$ below the pial surface) of nude mice. A total of 24 mice were used for the intracranial xenograft tumour model, six mice per group. TMZ was formulated in $3 \%$ DMSO, 50\% PEG300 and 0.5\% Tween 80 as suggested by Selleck Chemicals. Cells were implanted into brain of nude mice, and 14 days later, mice received intraperitoneal injections of TMZ $(40 \mathrm{mg} / \mathrm{kg} / \mathrm{d})$ or saline once a day for 7 days. Tumour growth was measured 45 days after tumour inoculation using an IVIS Lumina III In Vivo Imaging System.

\section{Immunohistochemistry}

GBM tumour and mouse paraffin sections were dewaxed, rehydrated and antigen retrieval was performed. Sections were blocked with $3 \%$ hydrogen peroxide for $10 \mathrm{~min}$ and with normal goat serum for $30 \mathrm{~min}$ at room temperature. Then, the sections were incubated with anti-LRRC4 (Abcam), DEPTOR (Cell Signaling Technology), BECN1 (Proteintech) and LC3B (Cell Signaling Technology) antibodies for $12 \mathrm{~h}$ at $4{ }^{\circ} \mathrm{C}$ and were incubated with biotinylated secondary antibody (Maxim Biotechnologies) for $30 \mathrm{~min}$ at room temperature and streptavidin conjugated HRP for $30 \mathrm{~min}$. Staining was visualized with 3,3-diaminobenzidine (DAB; Maxim Biotechnologies) and was counterstained with haematoxylin.

\section{Apoptosis measurement}

Apoptosis was assessed by labelling cells with annexin-VFITC and propidium iodide (PI). Cells were then analysed ( $n=20,000)$ by flow cytometry (Accuri C6 cytometer, Becton-Dickinson). Annexin V-positive cells (PI negative and positive) were considered apoptotic.

\section{Electron microscopy}

Cells were treated as indicated and fixed with $2.5 \%$ glutaraldehyde containing $0.1 \mathrm{~mol} / \mathrm{l}$ sodium cacodylate. 
Samples were fixed using $1 \%$ osmium tetroxide, followed by dehydration with an increasing concentration gradient of ethanol and propylene oxide. Samples were then embedded, cut into 50-nm sections, and stained with $3 \%$ uranyl acetate and lead citrate. Images were acquired using a JEM-1200 electron microscope (JEOL).

\section{Statistical analysis}

Data are presented as the mean \pm SD from at least three separate experiments, and the data were analysed with GraphPad Prism 5 (La Jolla, CA, USA). Differences between variables of two groups were examined by Student's $t$ test, and one-way ANOVA was used to evaluate the differences among variables of multiple groups. OS curves for the xenograft tumour model mice were calculated by the Kaplan-Meier method. The results were considered significant when $p<0.05$ was obtained.

\section{Data availability}

All authors ensure that all data generated or analysed during this study are included in this published article.

Funding This works was supported by the National Natural Science Foundation of China (Grant No. 81874150) and Graduate Student Research Innovation Project in Hunan Province (2019zzts084).

Author contributions WZ, QL, QL, MW conceived and designed the experiments. JF performed the main experiments and analysed the data. YZ participated in mice model preformation. XR, DL, HF and CL participated in cell survival and migration assay. JF and MW wrote the manuscript. All authors read and approved the final manuscript.

\section{Compliance with ethical standards}

Conflict of interest The authors declare that they have no conflict of interest.

Publisher's note Springer Nature remains neutral with regard to jurisdictional claims in published maps and institutional affiliations.

Open Access This article is licensed under a Creative Commons Attribution 4.0 International License, which permits use, sharing, adaptation, distribution and reproduction in any medium or format, as long as you give appropriate credit to the original author(s) and the source, provide a link to the Creative Commons license, and indicate if changes were made. The images or other third party material in this article are included in the article's Creative Commons license, unless indicated otherwise in a credit line to the material. If material is not included in the article's Creative Commons license and your intended use is not permitted by statutory regulation or exceeds the permitted use, you will need to obtain permission directly from the copyright holder. To view a copy of this license, visit http://creativecommons. org/licenses/by/4.0/.

\section{References}

1. Dunn GP, Rinne ML, Wykosky J, Genovese G, Quayle SN, Dunn IF, et al. Emerging insights into the molecular and cellular basis of glioblastoma. Genes Dev. 2012;26:756-84.

2. Ostrom QT, Gittleman H, Liao P, Rouse C, Chen Y, Dowling J, et al. CBTRUS statistical report: primary brain and central nervous system tumors diagnosed in the United States in 2007-11. Neuro Oncol. 2014;16(Suppl 4):v1-63.

3. Yu T, Wang X, Zhi T, Zhang J, Wang Y, Nie E, et al. Delivery of MGMT mRNA to glioma cells by reactive astrocyte-derived exosomes confers a temozolomide resistance phenotype. Cancer Lett. 2018;433:210-20.

4. Masui K, Cloughesy TF, Mischel PS. Review: molecular pathology in adult high-grade gliomas: from molecular diagnostics to target therapies. Neuropathological Soc. 2012;38:271-91.

5. Hombach-Klonisch S, Mehrpour M, Shojaei S, Harlos C, Pitz M, Hamai A, et al. Glioblastoma and chemoresistance to alkylating agents: involvement of apoptosis, autophagy, and unfolded protein response. Pharmacol Ther. 2018;184:13-41.

6. Murat A, Migliavacca E, Gorlia T, Lambiv WL, Shay T, Hamou $M$, et al. Stem cell-related "Self-Renewal" signature and high epidermal growth factor receptor expression associated with resistance to concomitant chemoradiotherapy in glioblastoma. J Clin Oncol. 2008;26:3015-24.

7. Mokarram P, Albokashy M, Zarghooni M, Moosavi MA, Sepehri $\mathrm{Z}$, Chen QM, et al. New frontiers in the treatment of colorectal cancer: Autophagy and the unfolded protein response as promising targets. Autophagy. 2017;13:781-819.

8. Johansen T, Lamark T. Selective autophagy mediated by autophagic adapter proteins. Autophagy. 2011;7:279-96.

9. Hale AN, Ledbetter DJ, Gawriluk TR, Rucker ER. Autophagy: regulation and role in development. Autophagy. 2013;9:951-72.

10. Cosin-Roger J, Simmen S, Melhem H, Atrott K, Frey-Wagner I, Hausmann M, et al. Hypoxia ameliorates intestinal inflammation through NLRP3/mTOR downregulation and autophagy activation. Nat Commun. 2017;8:98.

11. Ogata M, Hino S, Saito A, Morikawa K, Kondo S, Kanemoto S, et al. Autophagy is activated for cell survival after endoplasmic reticulum stress. Mol Cell Biol. 2006;26:9220-31.

12. Huang J, Lam GY, Brumell JH. Autophagy signaling through reactive oxygen species. Antioxid Redox Signal. 2011;14:2215-31.

13. Nixon RA. The role of autophagy in neurodegenerative disease. Nat Med. 2013;19:983-97.

14. Guo JY, Xia B, White E. Autophagy-mediated tumor promotion. Cell. 2013;155:1216-9.

15. White E. Deconvoluting the context-dependent role for autophagy in cancer. Nat Rev Cancer. 2012;12:401-10.

16. Jiang P, Mizushima N. Autophagy and human diseases. Cell Res. 2014;24:69-79.

17. Yu T, Guo F, Yu Y, Sun T, Ma D, Han J, et al. Fusobacterium nucleatum promotes chemoresistance to colorectal cancer by modulating autophagy. Cell. 2017;170:548-63.

18. Yang ZJ, Chee CE, Huang S, Sinicrope F. Autophagy modulation for cancer therapy. Cancer Biol Ther. 2011;11:169-76.

19. Ru WJ, Qian J, Li D, Ling LX, Chen T, Jiang LI, et al. Identification of LRRC4, a novel member of leucine-rich repeat (LRR) superfamily, and its expression analysis in brain tumor. Prog Biochem Biophysics. 2002;29:233-9.

20. Seho K, Alain B, Hye Sun C, Seok-Kyu K, Jooyeon W, Hyun Woo L, et al. NGL family PSD-95-interacting adhesion molecules regulate excitatory synapse formation. Nat Neurosci. 2006;9:1294-301.

21. Jooyeon W, Seok-Kyu K, Seungwon C, Seho K, Jae-Ran L, Dunah AW, et al. Trans-synaptic adhesion between NGL-3 and 
LAR regulates the formation of excitatory synapses. Nat Neurosci. 2009; $12: 428$.

22. Xu G, Wang R, Wang Z, Lei Q, Yu Z, Liu C, et al. NGL-2 is a new partner of PAR complex in axon differentiation. J Neurosci. 2015;35:7153-64.

23. Wu M, Huang C, Gan K, Huang H, Chen Q, Ouyang J, et al. LRRC4, a putative tumor suppressor gene, requires a functional leucine-rich repeat cassette domain to inhibit proliferation of glioma cells in vitro by modulating the extracellular signalregulated kinase/protein kinase $\mathrm{B} /$ nuclear factor-kappaB pathway. Mol Biol Cell. 2006;17:3534-42.

24. Li P, Xu G, Li G, Wu M. Function and mechanism of tumor suppressor gene LRRC4/NGL-2. Mol Cancer. 2014;13:266.

25. Wu M, Huang C, Li X, Li X, Gan K, Chen Q, et al. LRRC4 inhibits glioblastoma cell proliferation, migration, and angiogenesis by downregulating pleiotropic cytokine expression and responses. J Cell Physiol. 2008;214:65-74.

26. Minghua W, Qiong C, Dan L, Xiaoling L, Xiayu L, Chen H, et al. LRRC4 inhibits human glioblastoma cells proliferation, invasion, and proMMP-2 activation by reducing SDF-1 alpha/CXCR4-mediated ERK1/2 and Akt signaling pathways. $J$ Cell Biochem. 2010;103:245-55.

27. Wang Z, Guo Q, Wang R, Xu G, Li P, Sun Y, et al. The D Domain of LRRC4 anchors ERK1/2 in the cytoplasm and competitively inhibits MEK/ERK activation in glioma cells. J Hematol Oncol. 2016;9:130.

28. Li P, Feng J, Liu Y, Liu Q, Fan L, Liu Q, et al. Novel therapy for glioblastoma multiforme by restoring LRRC4 in tumor cells: LRRC4 inhibits tumor-infitrating regulatory $\mathrm{T}$ cells by cytokine and programmed cell death 1-containing exosomes. Front Immunol. 2017;8:1748.

29. Peterson TR, Laplante M, Thoreen CC, Sancak Y, Kang SA, Kuehl WM, et al. DEPTOR is an mTOR inhibitor frequently overexpressed in multiple myeloma cells and required for their survival. Cell. 2009;137:873-86.

30. Zhao Y, Xiong X, Sun Y. DEPTOR, an mTOR inhibitor, is a physiological substrate of $\mathrm{SCF}$ (betaTrCP) E3 ubiquitin ligase and regulates survival and autophagy. Mol Cell. 2011;44:304-16.

31. Zhang W, Rajan I, Savelieva KV, Wang CY, Vogel P, Kelly $\mathrm{M}$, et al. Netrin-G2 and netrin-G2 ligand are both required for normal auditory responsiveness. Genes Brain Behav. 2008;7:385-92.

32. Soto F, Zhao L, Kerschensteiner D. Synapse maintenance and restoration in the retina by NGL2. eLife. 2018;7:e30388.

33. Um SM, Ha S, Lee H, Kim J, Kim K, Shin W, et al. NGL-2 deletion leads to autistic-like behaviors responsive to NMDAR modulation. Cell Rep. 2018;23:3839-51.
34. Ghavami S, Shojaei S, Yeganeh B, Ande SR, Jangamreddy JR, Mehrpour M, et al. Autophagy and apoptosis dysfunction in neurodegenerative disorders. Prog Neurobiol. 2014;112:24-49.

35. Menzies FM, Fleming A, Caricasole A, Bento CF, Andrews SP, Ashkenazi A, et al. Autophagy and neurodegeneration: pathogenic mechanisms and therapeutic opportunities. Neuron. 2017;93:1015-34.

36. Nanegrungsunk D, Onchan W, Chattipakorn N, Chattipakorn SC. Current evidence of temozolomide and bevacizumab in treatment of gliomas. Neurological Res. 2015;37:167-83.

37. Wick W, Platten M. Understanding and targeting alkylator resistance in glioblastoma. Cancer Disco. 2014;4:1120-2.

38. Zhou W, Chen C, Shi Y, Wu Q, Gimple RC, Fang X, et al. Targeting glioma stem cell-derived pericytes disrupts the bloodtumor barrier and improves chemotherapeutic efficacy. Cell Stem Cell. 2017;21:591-603.

39. Yan Y, Xu Z, Dai S, Qian L, Sun L, Gong Z. Targeting autophagy to sensitive glioma to temozolomide treatment. J Exp Clin Cancer Res. 2016;35:23.

40. Hori YS, Hosoda R, Akiyama Y, Sebori R, Wanibuchi M, Mikami $\mathrm{T}$, et al. Chloroquine potentiates temozolomide cytotoxicity by inhibiting mitochondrial autophagy in glioma cells. J Neurooncol 2015;122:11-20.

41. Zanotto-Filho A, Braganhol E, Klafke K, Figueiro F, Terra SR, Paludo FJ, et al. Autophagy inhibition improves the efficacy of curcumin/temozolomide combination therapy in glioblastomas. Cancer Lett. 2015;358:220-31.

42. Dunlop EA, Tee AR. mTOR and autophagy: a dynamic relationship governed by nutrients and energy. Semin Cell Dev Biol. 2014;36:121-9.

43. Wang Z, Zhong J, Inuzuka H, Gao D, Shaik S, Sarkar FH, et al. An evolving role for DEPTOR in tumor development and progression. Neoplasia. 2012;14:368-75.

44. Duan S, Skaar JR, Kuchay S, Toschi A, Kanarek N, Ben-Neriah $\mathrm{Y}$, et al. mTOR generates an auto-amplification loop by triggering the betaTrCP- and CK1alpha-dependent degradation of DEPTOR. Mol Cell. 2011;44:317-24.

45. Chin SF, Wang Y, Thorne NP, Teschendorff AE, Pinder SE, Vias $\mathrm{M}$, et al. Using array-comparative genomic hybridization to define molecular portraits of primary breast cancers. Oncogene. 2007;26:1959-70.

46. Catena V, Fanciulli M. Deptor: not only a mTOR inhibitor. J Exp Clin Cancer Res. 2017;36:12.

47. Efeyan A, Sabatini DM. mTOR and cancer: many loops in one pathway. Curr Opin Cell Biol. 2010;22:169-76.

48. Wang Q, Zhou Y, Rychahou P, Harris JW, Zaytseva YY, Liu J, et al. Deptor is a novel target of Wnt/beta-Catenin/c-Myc and contributes to colorectal cancer cell growth. Cancer Res. 2018;78:3163-75. 